\title{
Systematic manipulation of the surface conductivity of $\mathrm{SmB}_{6}$
}

\author{
M. Victoria Ale Crivillero, ${ }^{1}$ M. König,${ }^{1}$ J. C. Souza $\odot,{ }^{2,1}$ P. G. Pagliuso,${ }^{2}$ J. Sichelschmidt $\odot,{ }^{1}$ \\ Priscila F. S. Rosa, ${ }^{3}$ Z. Fisk, ${ }^{4}$ and S. Wirth $0^{1, *}$ \\ ${ }^{1}$ Max-Planck-Institute for Chemical Physics of Solids, Nöthnitzer Strasse 40, 01187 Dresden, Germany \\ ${ }^{2}$ Instituto de Física “Gleb Wataghin,” UNICAMP, 13083-859 Campinas, São Paulo, Brazil \\ ${ }^{3}$ Los Alamos National Laboratory, Los Alamos, New Mexico 87545, USA \\ ${ }^{4}$ Department of Physics, University of California, Irvine, California 92697, USA
}

(Received 2 March 2021; revised 28 April 2021; accepted 12 May 2021; published 1 June 2021)

\begin{abstract}
We show that the resistivity plateau of $\mathrm{SmB}_{6}$ at low temperature, typically taken as a hallmark of its conducting surface state, can systematically be influenced by different surface treatments. We investigate the effect of inflicting an increasing number of handmade scratches and microscopically defined focused ion beam-cut trenches on the surfaces of flux-grown $\mathrm{Sm}_{1-x} \mathrm{Gd}_{x} \mathrm{~B}_{6}$ with $x=0$ and 0.0002 . Both treatments increase the resistance of the low-temperature plateau, whereas the bulk resistance at higher temperatures largely remains unaffected. Notably, the temperature at which the resistance deviates from the thermally activated behavior decreases with cumulative surface damage. These features are more pronounced for the focused ion beam treated samples, with the difference likely being related to the absence of microscopic defects such as subsurface cracks. Therefore, our method presents a systematic way of controlling the surface conductance.
\end{abstract}

DOI: 10.1103/PhysRevResearch.3.023162

\section{INTRODUCTION}

Over the past decade, the proposed topological Kondo insulator $\mathrm{SmB}_{6}$ has seen a surge of research interest [1] despite its more than half-a-century-old history [2,3]. This interest stems from a combination of complex correlated electron physics and the proposed topologically nontrivial surface states resulting from spin-orbit-driven band inversion in the bulk [4-6].

Irrespective of the direct involvement of the surface in the topical physics, relatively little is known about its properties. While the bulk of $\mathrm{SmB}_{6}$ is known for its intermediate and temperature-dependent $\mathrm{Sm}$ valence of approximately 2.6 at low temperature $[2,7-10]$, the valence at the surface appears to be closer to $3+[9,10]$. This change in valence could be related to the formation of $\mathrm{Sm}_{2} \mathrm{O}_{3}$ near the surface, resulting from an oxidation of the near-surface $\mathrm{Sm}$. A changed surface chemistry may also shift the chemical potential at the surface [11] and may lead to time-dependent surface properties [12]. Consequently, in numerous studies relying on highly surfacesensitive techniques such as scanning tunneling microscopy and spectroscopy (STM and STS) [13-19] and angle-resolved photoemission spectroscopy (ARPES) [20-26], $\mathrm{SmB}_{6}$ surfaces were prepared by in situ cleaving in ultrahigh vacuum

\footnotetext{
*Steffen.Wirth@cpfs.mpg.de

Published by the American Physical Society under the terms of the Creative Commons Attribution 4.0 International license. Further distribution of this work must maintain attribution to the author(s) and the published article's title, journal citation, and DOI. Open access publication funded by the Max Planck Society.
}

(UHV) conditions. However, $\mathrm{SmB}_{6}$ is difficult to break, and surfaces perpendicular to the main crystallographic axes of the cubic structure (space group $P m \overline{3} m$ ) are polar in nature, often giving rise to $(2 \times 1)$ reconstructed surfaces. Notably, even cleaved surfaces may exhibit valence inhomogeneities [27] and band-bending effects [26,28,29].

Also for cases of less surface-sensitive techniques, such as resistivity measurements, surfaces often need to be prepared, e.g., by polishing or etching (see, e.g., Refs. [30-38]). However, such surface preparation may influence the surface itself, e.g., by disrupting the crystal structure at the surface, introducing impurities, or, again, changing the $\mathrm{Sm}$ valence. One particularly interesting example here is the creation of so-called subsurface cracks by rough polishing [39]. These subsurface cracks constitute additional surfaces with their own surface states, which conduct in parallel to the actual sample surface. Hence, care has to be taken when comparing different results since differences in the applied surface preparation procedure may result in differences in the measured properties. To make things more complicated, there can also be differences between samples grown by either the floating zone or Al flux technique, not only intrinsically [38,40-43] but also with respect to the impact of surface preparation as shown exemplary for etched surfaces [38].

We here apply a systematic way of manipulating the sample surface by utilizing a focused ion beam (FIB), complemented by a rather crude surface scratching. The low-temperature resistivity plateau of our flux-grown $\mathrm{SmB}_{6}$ samples, typically taken as a hallmark of the conducting surface state, can be influenced considerably, yet consistently, by both surface treatments, indicating impaired surface states. As expected, the thermally activated transport across the bulk gap is not affected significantly by the surface treatments. 


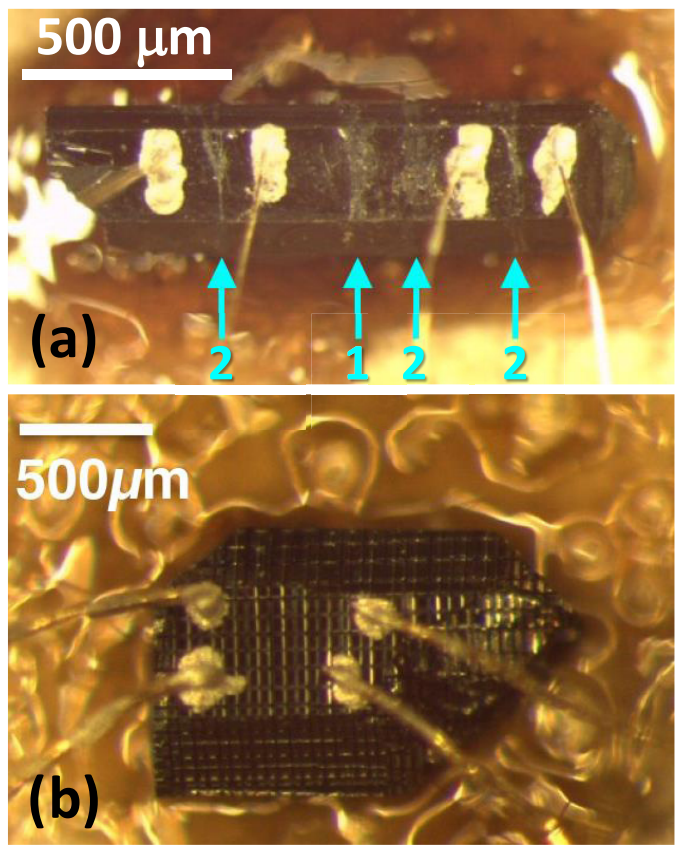

FIG. 1. Images of two exemplary $\mathrm{SmB}_{6}$ samples. (a) Optical microscopy image of a surface with scratches between the glued-on contacts. The scratches inflicted in the first (1) and second (2) run are marked by arrows. (b) Optical image of a sample with lines cut by FIB (after six runs) and with contacts attached.

\section{EXPERIMENT}

The samples $\mathrm{Sm}_{1-x} \mathrm{Gd}_{x} \mathrm{~B}_{6}$ used in this study were grown by the Al flux technique [44] with a Gd content of $x=0$ and 0.0002 . The tiny amount of Gd for the latter samples was confirmed by magnetic susceptibility measurements. It allowed electron spin resonance (ESR) measurements, which will be reported elsewhere [45]. We did not observe any noticeable differences in the here reported properties of samples with $x=0$ and 0.0002 . Therefore, we concentrate in the following on samples $x=0.0002$ which were studied more extensively. Energy-dispersive x-ray (EDX) spectroscopy conducted within our FIB equipment at pressures in the $10^{-6}$ mbar range did not show any elements other than Sm, B, O, and Al, with Gd being below the detection limit. Upon using the FIB to remove a layer of a few $\mu \mathrm{m}$ thickness, the Al signal is no longer detectable within these sputtered areas.

As a crude way of disrupting the surface we cut lines by means of a diamond scribe. Because of the hardness of $\mathrm{SmB}_{6}$, considerable force had to be applied to inflict the line damage to the sample surface as shown in Fig. 1(a). In this example, the first scratch (marked by an arrow and a number) was only applied to the front surface. In a second step, more scratches were applied, and all scratches now cover also the back and side surfaces to form closed rings approximately perpendicular to the long sample axis. In a third step, the scratches were deepened by applying more force to the diamond scribe. Figure 1(a) was taken subsequent to the second scratching and with contacts for resistance measurements attached.

In an effort to structure the surfaces of our samples in a much more systematic and controlled fashion, we utilized a
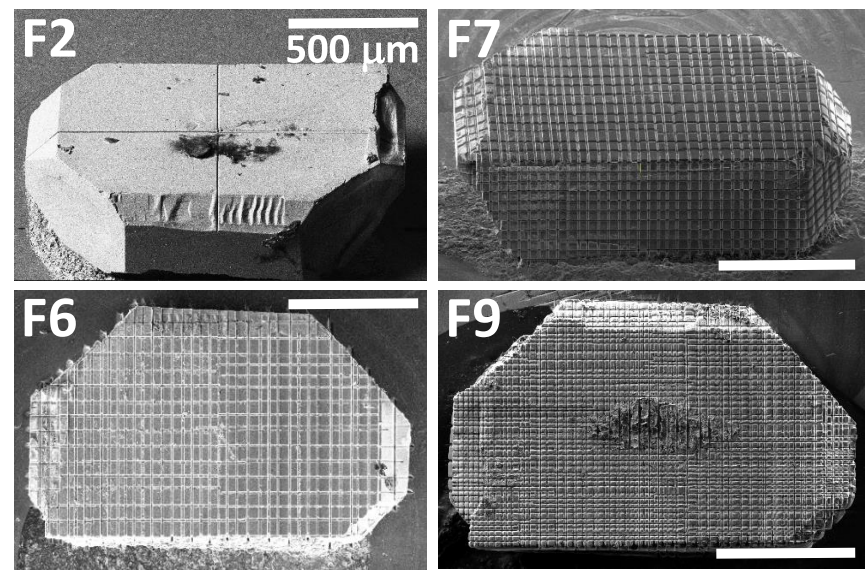

FIG. 2. SEM images of an $\mathrm{SmB}_{6}$ sample after FIB cutting an increasing number of lines. Shown are examples after FIB runs F2, F6, F7, and F9. The dark patches at the centers of F2 and F9 are residue of paraffin used for fixing the sample. All scale bars: $500 \mu \mathrm{m}$.

FIB. Trenches of about 7-10 $\mu \mathrm{m}$ in depth were cut by Xe ions at beam currents of $500 \mathrm{nA}$ with an acceleration voltage of $30 \mathrm{kV}$ in consecutive runs. In a first run (denoted F1 in the following), a single line is cut across the middle of the sample, dividing the surface in two parts. In a second run (F2), each half is subdivided into two fields of similar size by cutting a line perpendicular to the first one; the resulting crossed lines are seen in the scanning electron microscopy (SEM) image F2 in Fig. 2. In subsequent runs, the number of lines is about doubled in each direction by cutting additional lines approximately parallel to the already existing ones. The resulting grids of FIB-cut lines after selected runs are presented in Fig. 2. In the case of this particular sample, during runs F1-F6 cuts were inflicted only on the front and back surface (the front surface is seen in F6 and F9 in Fig. 2). Run F7 was used to transfer the existing grid to all side surfaces (see F7 in Fig. 2). Consecutive runs F8 and F9 included the front and back surfaces. After the final run (F9), this sample was FIB cut to a minimum grid line distance of about $15 \mu \mathrm{m}$. We note that this distance is still large compared to the effective carrier mean free path $\ell<1 \mu \mathrm{m}[45,46]$.

Resistance measurements were usually conducted after some FIB runs or line scratches, using a physical property measurement system (PPMS) by Quantum Design. In the case of FIB-cut sample surfaces, van der Pauw-type measurements were conducted. A sample (different from the one presented in Fig. 2) after FIB run F6 with contacts attached is shown in Fig. 1(b).

\section{RESULTS}

\section{A. $\mathrm{Sm}_{1-x} \mathbf{G d}_{x} \mathbf{B}_{6}$ samples with scratched surfaces}

Resistances of the sample shown in Fig. 1(a) before and after inflicting an increasing number of scratches to the sample surface are presented in Fig. 3(a). Clearly, the first scratch did not significantly change the resistance, possibly because the first scratch did not form a closed ring around the sample. Consecutive scratches formed closed rings and introduced resistance changes. These changes, however, are exclusively 

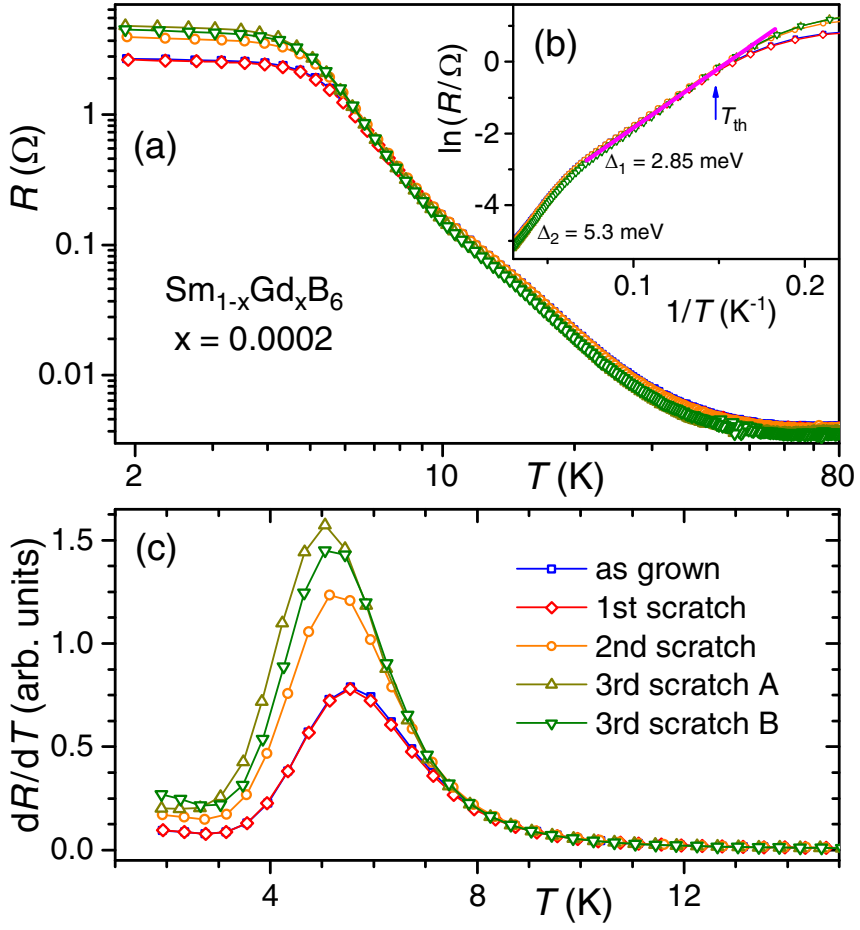

FIG. 3. (a) Double-logarithmic plot of the resistance of a $\mathrm{Sm}_{1-x} \mathrm{Gd}_{x} \mathrm{~B}_{6}$ sample with $x=0.0002$ before, and subsequent to, an increasing number of scratches. After the third scratching, resistances were measured through two sets of contacts, A and B. (b) $\ln R$ vs $1 / T$ representation of the intermediate-temperature data. The magenta line illustrates thermally activated behavior, from which $R(T)$ deviates at $T_{\text {th }}$ (shown for the as-grown sample). (c) Derivative of the low- $T$ resistance as a function of temperature.

limited to the low-temperature regime, as shown in the different representations in Figs. 3(a)-3(c). This finding is in agreement with the fact that the resistance at higher $T$ reflects bulk properties while the surface state dictates the resistance behavior only at $T$ below a few $\mathrm{K}$. The bulk hybridization gap $\Delta$ can be estimated from $R(T) \propto \exp \left(\Delta / k_{\mathrm{B}} T\right)$, where $k_{\mathrm{B}}$ is the Boltzmann constant. Typically, for pure $[36,47]$ and slightly Gd-substituted $[17,48] \mathrm{SmB}_{6}$ two regimes with different gap values are observed depending on the $T$ range considered. This also holds for our measurements with $\Delta_{1}=2.85( \pm 0.07) \mathrm{meV}$ and $\Delta_{2}=5.3( \pm 0.1) \mathrm{meV}$, independent of the scratches [see Fig. 3(b)]. However, the scratches do influence the lower bound $T_{\text {th }}$ of the temperature range within which $R(T)$ can be described by thermally activated behavior [the latter is marked by a magenta line in Fig. 3(b)]. Obviously, there is a clear trend: The more pronounced the scratches, the lower is $T_{\mathrm{th}}$. We find for the as-grown sample and after the first scratch $T_{\mathrm{th}} \approx 7 \mathrm{~K}$ [see the arrow in Fig. 3(b)], after the second scratching $T_{\mathrm{th}} \approx 6.4 \mathrm{~K}$, and $T_{\mathrm{th}} \approx 6.1 \mathrm{~K}$ after the third scratching. This trend is also seen in the derivative $d R / d T$ in Fig. 3(c). As outlined in Ref. [36], the thermally activated behavior, i.e., the exponential increase of $R(T)$, is a clear hallmark of the bulk resistance, which is superseded by the additional surface component upon lowering $T$ assuming a parallel conductance model $[31,33,49]$. The low- $T$ resistance plateau indicates the presence of the surface states even after scratching. Yet, based on the trend of $T_{\text {th }}$, the crossover
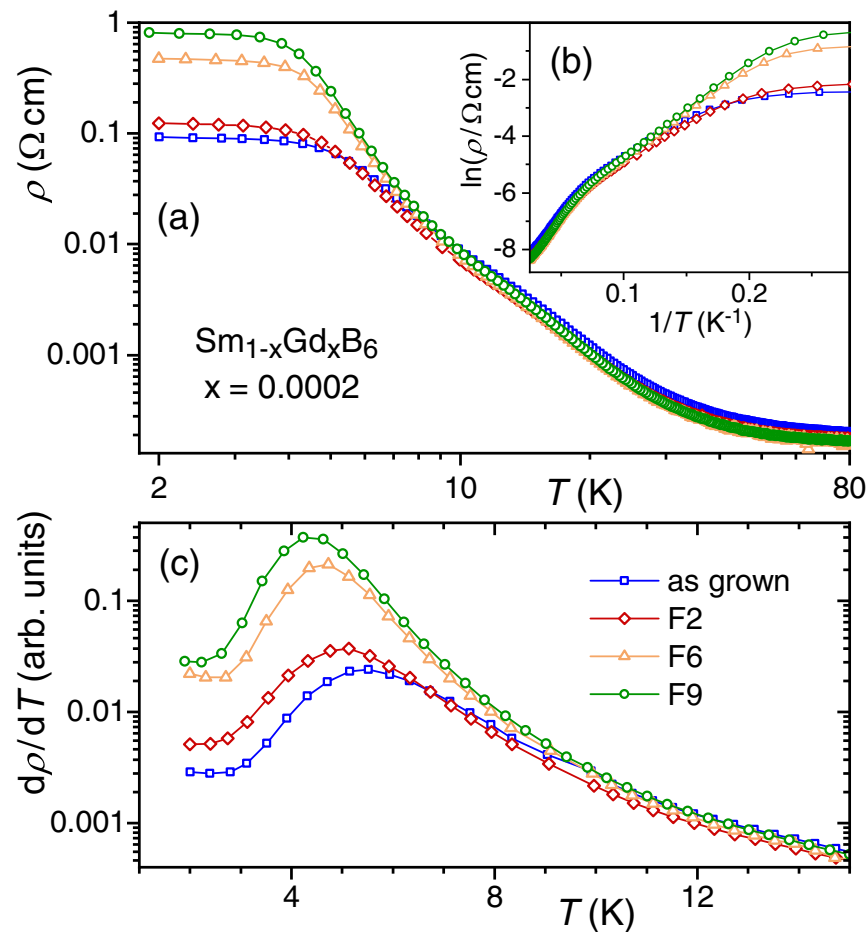

FIG. 4. (a) Double-logarithmic plot of the resistivity $\rho$ of $\mathrm{Sm}_{1-x} \mathrm{Gd}_{x} \mathrm{~B}_{6}$ with $x=0.0002$ before and after FIB cutting an increasing number of lines (see Fig. 2). (b) $\ln \rho$ vs $1 / T$ representation of the intermediate-temperature data. (c) Low- $T$ range of the derivative $d \rho / d T$.

from bulk-dominated conductivity (roughly above $10 \mathrm{~K}$ ) to surface-dominated conductivity (below about $3 \mathrm{~K}$ ) appears to take place at a lower temperature. The increased value of the low- $T$ resistance plateau measured on the damaged surfaces could be caused by either a decreased conductivity of the intrinsic surface state or an additional damage layer at the scratched areas below which the intrinsic surface state reconstructs (or a combination thereof). However, the surface state still develops and appears to govern the $R(T)$ below a similar $T \approx 3 \mathrm{~K}$ at which the $R(T)$ slope no longer changes [see Fig. 3(c)]. Here, we note that subsequent to the measurement "3rd scratch A" the contacts were completely removed and attached anew for measurement "B," showing that the contacts themselves have no significant influence on $R(T)$. In particular, the difference of the $R(T)$ values at low $T$ is less than $7 \%$ (compared to the $\sim 20 \%$ change between the second and third scratch).

\section{B. FIB-cut trenches on sample surfaces}

In order to manipulate the sample surface in a much more controlled and systematic way, we also measured the resistivity of FIB-treated samples as described above. Exemplary resistivity data of a sample $\mathrm{Sm}_{1-x} \mathrm{Gd}_{x} \mathrm{~B}_{6}$ with $x=0.0002$ are given in Fig. 4(a) for an increasing number of FIB-cut lines on its surfaces. We note that the contacts needed to be removed before each subsequent FIB run. Although great care was taken to reattach the contacts after the FIB run at the very same positions, a marginal influence on the resistivity values cannot 
TABLE I. Fit parameters for the two thermally activated gaps of the FIB-cut sample shown in Fig. 2, obtained from the resistivity data of Fig. 4. The gap values $\Delta$ and the temperature ranges within which the fits hold are given. $d$ denotes the approximate distance between FIB-cut lines (or the lines and the sample perimeter in the case of F2).

\begin{tabular}{|c|c|c|c|c|c|c|}
\hline \multirow[b]{2}{*}{ Sample } & \multirow[b]{2}{*}{$\begin{array}{c}d \\
(\mu \mathrm{m})\end{array}$} & \multicolumn{3}{|c|}{ Gap at lower $T$} & \multicolumn{2}{|c|}{ Gap at higher $T$} \\
\hline & & $\begin{array}{c}\Delta_{1} \\
(\mathrm{meV})\end{array}$ & $\begin{array}{l}T_{\text {th }} \\
(\mathrm{K})\end{array}$ & $\begin{array}{l}\text { Upper } T \\
\text { (K) }\end{array}$ & $\begin{array}{c}\Delta_{2} \\
(\mathrm{meV})\end{array}$ & $\begin{array}{c}\text { Fitted } T \text { range } \\
(\mathrm{K})\end{array}$ \\
\hline As grown & & 2.4 & 7.1 & 12.3 & 5.2 & $18.4-29.2$ \\
\hline $\mathrm{F} 2$ & 700 & 2.4 & 6.3 & 12.3 & 5.2 & $18.7-29.4$ \\
\hline F6 & 40 & 2.8 & 5.4 & 12.3 & 5.3 & $18.8-30.3$ \\
\hline F9 & 15 & 2.9 & 5.1 & 12.4 & 5.4 & $18.6-29.4$ \\
\hline Error & & \pm 0.2 & \pm 0.3 & \pm 0.4 & \pm 0.2 & \pm 0.8 \\
\hline
\end{tabular}

be excluded entirely. Due to the small size and position of the contacts, there was no conducting path between contacts uninterrupted by FIB-cut lines-even via side surfaces-after FIB run F5 already [as can be inferred from Fig. 1(b) for the second sample].

Already the first cross of FIB-cut lines (F2) increases the $\rho(T)$ values within the plateau at low $T$ compared to the as-grown surface by more than $30 \%$, which appears to be well beyond the geometry inaccuracy. Interestingly, the reduction of $T_{\text {th }}$ after FIB run F2 is very similar to those after the second and third scratch, i.e., for a comparable number of lines and scratches. Upon increasing the FIB-cut line density, the low- $T$ resistivity increases further such that $\rho(T)$ of F9 at low temperature exceeds the value of the as-grown surface by almost an order of magnitude. Just as pronounced is the concomitant drop of $T_{\text {th }}$ by $2 \mathrm{~K}$ from as grown to F9 (see Table I). Most other parameters remain largely unaffected by the FIB surface structuring, and only $\Delta_{1}$ appears to be slightly modified. Nonetheless, the bulk sample properties remain essentially unaltered by the FIB treatment. Very similar trends were observed on a second FIB-cut sample, shown in Fig. 1(b).

The increased resistivities upon damaging the sample surfaces are in contrast to the observed decrease for substituted or intentionally imperfect samples [17,50-54] or ion-irradiated samples [55]. This might imply that our surface treatments by FIB or scratching do not influence the surfaces on the whole, but rather act on the surface states locally. On the other hand, an increased slope of the low- $T$ resistivity appears to generally indicate a diminished surface state.

\section{DISCUSSION}

In Ref. [39], the influence of subsurface cracks on the total resistivity is discussed. Such subsurface cracks provide additional conduction channels, and a decreased low- $T$ resistance upon surface scratching was reported [39]. Subsurface cracks could also be found below our scratched surface areas [see the arrow marks in Fig. 5(a)]. We note that such subsurface cracks could so far be found exclusively underneath the scratches, presumably indicating that the unscratched sample regions (including the pristine samples) are free of such subsurface
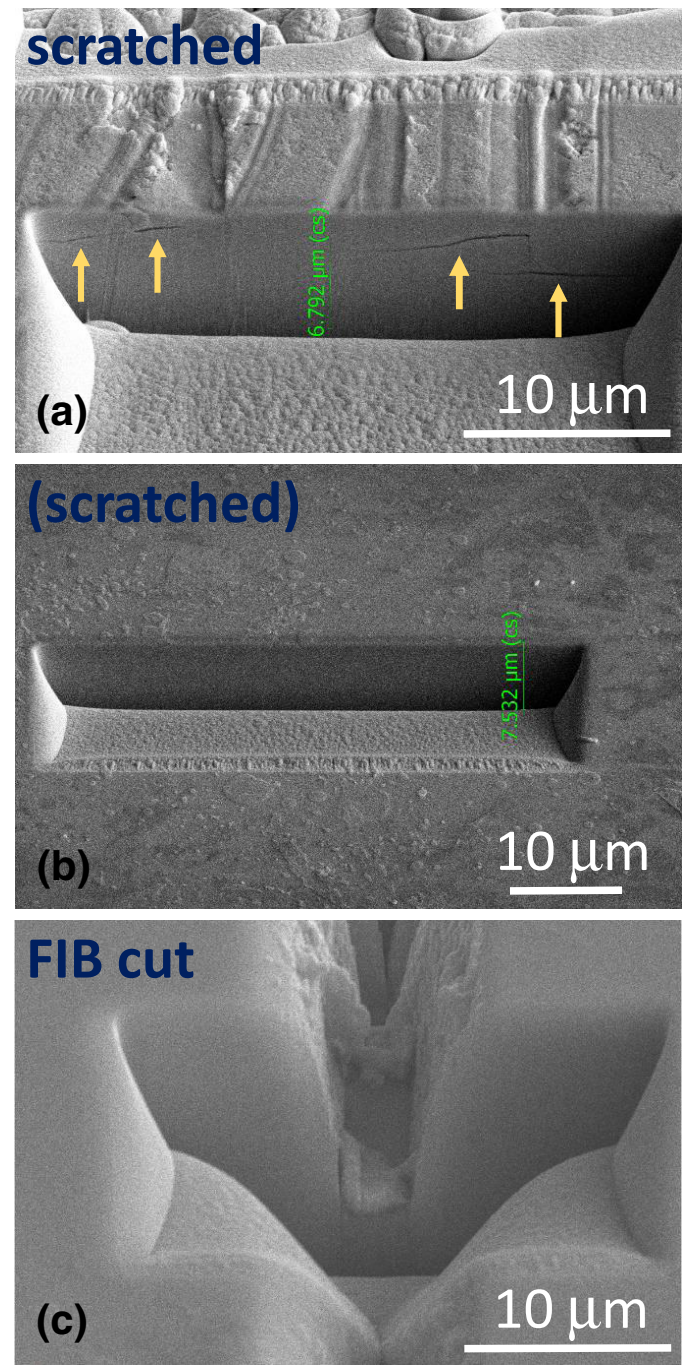

FIG. 5. Exemplary SEM images of (a) a sample cross section underneath a scratched line. Cracks are marked by arrows. (b) Same sample as in (a) but the cross section is taken at an area away from any scratch. (c) Cross section surrounding a FIB-cut line.

cracks [Fig. 5(b)]. The subsurface cracks can be found down to a few micrometers below the scratched surface. However, in contrast to the earlier findings [39], the sample resistance increases with scratching in our case [Fig. 3(a)]. Here, we recall that our scratches encircle the whole surface without leaving any possible current path on the surface untouched. Therefore, our approach seems to emphasize the impact of the surface conductance to the total sample resistance compared to Corbino-type measurements [32,36,39]. We therefore infer that the value of the low- $T$ resistance plateau is the result of two counteracting effects: While the subsurface cracks lower this value by introducing additional conductance channels, the surface conductance itself is hampered due to scratching, as also indicated by the lower $T_{\text {th }}$ values. In this respect, the intermediate- and high-temperature resistance regime provides important hints of largely unchanged bulk properties.

This picture is corroborated by the results of the FIB-cut samples. Although the trenches inflicted by the FIB cut deeper 
into the sample (up to about $10 \mu \mathrm{m}$ ) compared to the scratches (typically a few micrometers, with depths up to about $5 \mu \mathrm{m}$ ), we so far did not find any indication of subsurface cracks on FIB-treated samples [see the example in Fig. 5(c)]. The material directly at the bottom of the FIB-cut trenches, and to a lesser extent also at the sidewalls, is typically turned amorphous to a depth of several tens of nanometers and, in the case of preferential sputtering, nonstoichiometric [56]. Below this affected layer, the crystal structure is usually well preserved, with only occasional lattice defects caused by the ion bombardment. In this sense, the FIB treatment can be considered a controlled and systematic way of manipulating the surface conductance. The low- $T$ saturation of $\rho(T)$ [Fig. 4(a)] indicates that the conducting surface layer, albeit possibly encumbered, is still subsisting. We note that in one case we also conducted an abrasion of the whole sample surface of about $3 \mu \mathrm{m}$ deep by rastering the entire sample surface with the ion beam in a last run (i.e., after FIB cutting a line grid) and still observed indications of the surface layer, in line with Ref. [57]. This finding has an interesting consequence: The above-mentioned amorphous layer then covers the whole sample surface. Since this layer very likely prevents a surface reconstruction from forming, we can in all likelihood rule out a $2 \times 1$ surface reconstruction (as, e.g., observed in some cases by STM $[13,14,19]$ ) causing the conducting surface states. Also, the polarity change at the interface between amorphous and crystalline $\mathrm{SmB}_{6}$ is certainly smaller compared to a pure $\mathrm{SmB}_{6}$ surface. In consequence, all this makes conducting surface states driven by a nontrivial topology of the crystalline $\mathrm{SmB}_{6}$ more likely.

There are at least two contributions which may cause the increase of $\rho(T)$ at low $T$ upon FIB-cutting trenches: (i) The surface state may be tampered with and (ii) the surface area increases with the number of lines. The latter, however, appears not to be a decisive factor as an increased $\rho(T)$ is already observed for a small number of lines. As an example, the surface area of surface F6 in Fig. 4 is less than doubled compared to the as-grown one, but $\rho(2 \mathrm{~K})$ increased by a factor of more than 5. This strong increase of $\rho(T)$ at low temperature, along with the concomitantly lowered $T_{\text {th }}$ (Table I) as well as preliminary ESR results [45] suggest a reduction of the contribution of the surface states to the sample conductivity, possibly due to an FIB-induced depletion of the surface states. This might be related to a confinement of the surface states. In addition, disorder effects (in the bulk or/and near the surface due to our treatments) can be important in Kondo insulators as disorder can greatly affect the hybridization gap $[58,59]$ and, in turn, the surface states. As one example, the Sm valence near the surface can be modified [27,37], which could introduce changes to the surface conductivity.

As mentioned above, it is unlikely, and also not seen in our attempts, that the FIB treatment induces subsurface cracks. On the other hand, similar to the case of scratched sample surfaces, the surface state appears to be tampered with. Therefore, one may speculate that the relatively small increase of the low- $T$ resistance for the scratched surfaces compared to the FIB-treated ones is related to the subsurface cracks in the former. Of course, the severity of the inflicted damage to the respective surface may also differ.

\section{CONCLUSION}

We showed that introducing localized damage to the $\mathrm{SmB}_{6}$ surface by different treatments, such as mechanical surface scratching and FIB-cut trenches, can alter the low-temperature resistance plateau significantly. We find that the measured low-temperature $R$ value depends sensitively on the type of surface treatment and the structural damage incurred. In our cases, the bulk resistivity at higher temperatures remains largely unchanged, and hence the ratio between the resistances at high and at lowest temperature is not a good measure of the sample quality. However, the low-temperature limit to which the resistance follows a thermally activated behavior is found to be related to the severity of damage inflicted to the surface.

More generally, the systematic and well-controlled surface treatment by FIB as presented here may provide a path for the modification and patterning of surface states as recently suggested theoretically [60].

\section{ACKNOWLEDGMENTS}

J.C.S. acknowledges support through the RIA Doctorate funding by FAPESP, Brazil (Grants No. 2020/12283-0, No. 2018/11364-7, and No. 2017/10581-1). Work at Los Alamos National Laboratory was performed under the auspices of the U.S. Department of Energy, Office of Basic Energy Sciences, Division of Materials Science and Engineering. Z.F. acknowledges support from the LANL G. T. Seaborg Institute.
[1] L. Li, K. Sun, C. Kurdak, and J. W. Allen, Emergent mystery in the Kondo insulator samarium hexaboride, Nat. Rev. Phys. 2, 463 (2020).

[2] E. E. Vainshtein, S. M. Blokhin, and Y. B. Paderno, X-ray spectral investigation of samarium hexaboride, Sov. Phys.-Solid State 6, 2318 (1965).

[3] A. Menth, E. Buehler, and T. H. Geballe, Magnetic and Semiconducting Properties of $\mathrm{SmB}_{6}$, Phys. Rev. Lett. 22, 295 (1969).

[4] M. Dzero, K. Sun, V. Galitski, and P. Coleman, Topological Kondo Insulators, Phys. Rev. Lett. 104, 106408 (2010).

[5] T. Takimoto, $\mathrm{SmB}_{6}$ : A promising candidate for a topological insulator, J. Phys. Soc. Jpn. 80, 123710 (2011).
[6] M. Dzero, J. Xia, V. Galitski, and P. Coleman, Topological Kondo insulators, Annu. Rev. Condens. Matter Phys. 7, 249 (2016).

[7] J. M. Tarascon, Y. Isikawa, B. Chevalier, J. Etourneau, P. Hagenmuller, and M. Kasaya, Temperature dependence of the samarium oxidation state in $\mathrm{SmB}_{6}$ and $\mathrm{Sm}_{1-x} \mathrm{La}_{x} \mathrm{~B}_{6}$, J. Phys. (Paris) 41, 1141 (1980).

[8] M. Mizumaki, S. Tsutsui, and F. Iga, Temperature dependence of $\mathrm{Sm}$ valence in $\mathrm{SmB}_{6}$ studied by x-ray absorption spectroscopy, J. Phys.: Conf. Ser. 176, 012034 (2009).

[9] P. Lutz, M. Thees, T. R. F. Peixoto, B. Y. Kang, B. K. Cho, C.-H. Min, and F. Reinert, Valence characterisation of the subsurface region in $\mathrm{SmB}_{6}$, Philos. Mag. 96, 3307 (2016). 
[10] Y. Utsumi, D. Kasinathan, K.-T. Ko, S. Agrestini, M. W. Haverkort, S. Wirth, Y.-H. Wu, K.-D. Tsuei, D.-J. Kim, Z. Fisk, A. Tanaka, P. Thalmeier, and L. H. Tjeng, Bulk and surface electronic properties of $\mathrm{SmB}_{6}$ : A hard x-ray photoelectron spectroscopy study, Phys. Rev. B 96, 155130 (2017).

[11] W. K. Park, L. Sun, A. Noddings, D.-J. Kim, Z. Fisk, and L. H. Greene, Topological surface states interacting with bulk excitations in the Kondo insulator $\mathrm{SmB}_{6}$ revealed via planar tunneling spectroscopy, Proc. Natl. Acad. Sci. USA 113, 6599 (2016).

[12] Z.-H. Zhu, A. Nicolaou, G. Levy, N. P. Butch, P. Syers, X. F. Wang, J. Paglione, G. A. Sawatzky, I. S. Elfimov, and A. Damascelli, Polarity-Driven Surface Metallicity in $\mathrm{SmB}_{6}$, Phys. Rev. Lett. 111, 216402 (2013).

[13] M. M. Yee, Y. He, A. Soumyanarayanan, D.-J. Kim, Z. Fisk, and J. E. Hoffman, Imaging the Kondo insulating gap on $\mathrm{SmB}_{6}$, arXiv:1308.1085.

[14] S. Rößler, T.-H. Jang, D. J. Kim, L. H. Tjeng, Z. Fisk, F. Steglich, and S. Wirth, Hybridization gap and Fano resonance in $\mathrm{SmB}_{6}$, Proc. Natl. Acad. Sci. USA 111, 4798 (2014).

[15] W. Ruan, C. Ye, M. Guo, F. Chen, X. Chen, G.-M. Zhang, and Y. Wang, Emergence of a Coherent In-Gap State in the $\mathrm{SmB}_{6}$ Kondo Insulator Revealed by Scanning Tunneling Spectroscopy, Phys. Rev. Lett. 112, 136401 (2014).

[16] L. Jiao, S. Rößler, D. J. Kim, L. H. Tjeng, Z. Fisk, F. Steglich, and S. Wirth, Additional energy scale in $\mathrm{SmB}_{6}$ at low temperature, Nat. Commun. 7, 13762 (2016).

[17] L. Jiao, S. Rößler, D. Kasinathan, P. F. S. Rosa, C. Guo, H. Yuan, C.-X. Liu, Z. Fisk, F. Steglich, and S. Wirth, Magnetic and defect probes of the $\mathrm{SmB}_{6}$ surface state, Sci. Adv. 4, eaau4886 (2018).

[18] Z. Sun, A. Maldonado, W. S. Paz, D. S. Inosov, A. P. Schnyder, J. J. Palacios, N. Y. Shitsevalova, V. B. Filipov, and P. Wahl, Observation of a well-defined hybridization gap and in-gap states on the $\mathrm{SmB}_{6}(001)$ surface, Phys. Rev. B 97, 235107 (2018).

[19] H. Pirie, Y. Liu, A. Soumyanarayanan, P. Chen, Y. He, M. M. Yee, P. F. S. Rosa, J. D. Thompson, D.-J. Kim, Z. Fisk, X. Wang, J. Paglione, D. K. Morr, M. H. Hamidian, and J. E. Hoffman, Imaging emergent heavy Dirac fermions of a topological Kondo insulator, Nat. Phys. 16, 52 (2020).

[20] J. Jiang, S. Li, T. Zhang, Z. Sun, F. Chen, Z. R. Ye, M. Xu, Q. Q. Ge, S. Y. Tan, X. H. Niu, M. Xia, B. P. Xie, Y. F. Li, X. H. Chen, H. H. Wen, and D. L. Feng, Observation of possible topological in-gap surface states in the Kondo insulator $\mathrm{SmB}_{6}$ by photoemission, Nat. Commun. 4, 3010 (2013).

[21] E. Frantzeskakis, N. de Jong, B. Zwartsenberg, Y. K. Huang, Y. Pan, X. Zhang, J. X. Zhang, F. X. Zhang, L. H. Bao, O. Tegus, A. Varykhalov, A. de Visser, and M. S. Golden, Kondo Hybridization and the Origin of Metallic States at the (001) Surface of $\mathrm{SmB}_{6}$, Phys. Rev. X 3, 041024 (2013).

[22] J. D. Denlinger, J. W. Allen, J.-S. Kang, K. Sun, B.-I. Min, D.-J. Kim, and Z. Fisk, $\mathrm{SmB}_{6}$ photoemission: Past and present, JPS Conf. Proc. 3, 017038 (2014).

[23] N. Xu, P. K. Biswas, J. H. Dil, R. S. Dhaka, G. Landolt, S. Muff, C. E. Matt, X. Shi, N. C. Plumb, M. Radović, E. Pomjakushina, K. Conder, A. Amato, S. V. Borisenko, R. Yu, H. M. Weng, Z. Fang, X. Dai, J. Mesot, H. Ding et al., Direct observation of the spin texture in $\mathrm{SmB}_{6}$ as evidence of the topological Kondo insulator, Nat. Commun. 5, 4566 (2014).
[24] S. Suga, K. Sakamoto, T. Okuda, K. Miyamoto, K. Kuroda, A. Sekiyama, J. Yamaguchi, H. Fujiwara, A. Irizawa, T. Ito, S. Kimura, T. Balashov, W. Wulfhekel, S. Yeo, F. Iga, and S. Imada, Spin-polarized angle-resolved photoelectron spectroscopy of the so-predicted Kondo topological insulator $\mathrm{SmB}_{6}$, J. Phys. Soc. Jpn. 83, 014705 (2014).

[25] P. Hlawenka, K. Siemensmeyer, E. Weschke, A. Varykhalov, J. Sánchez-Barriga, N. Y. Shitsevalova, A. V. Dukhnenko, V. B. Filipov, S. Gabáni, K. Flachbart, O. Rader, and E. D. L. Rienks, Samarium hexaboride is a trivial surface conductor, Nat. Commun. 9, 517 (2018).

[26] C. E. Matt, H. Pirie, A. Soumyanarayanan, Y. He, M. M. Yee, P. Chen, Y. Liu, D. T. Larson, W. S. Paz, J. J. Palacios, M. H. Hamidian, and J. E. Hoffman, Consistency between ARPES and STM measurements on $\mathrm{SmB}_{6}$, Phys. Rev. B 101, 085142 (2020).

[27] V. B. Zabolotnyy, K. Fürsich, R. J. Green, P. Lutz, K. Treiber, C.-H. Min, A. V. Dukhnenko, N. Y. Shitsevalova, V. B. Filipov, B. Y. Kang, B. K. Cho, R. Sutarto, F. He, F. Reinert, D. S. Inosov, and $\mathrm{V}$. Hinkov, Chemical and valence reconstruction at the surface of $\mathrm{SmB}_{6}$ revealed by means of resonant soft x-ray reflectometry, Phys. Rev. B 97, 205416 (2018).

[28] M. Neupane, N. Alidoust, S.-Y. Xu, T. Kondo, Y. Ishida, D. J. Kim, C. Liu, I. Belopolski, Y. J. Jo, T.-R. Chang, H.-T. Jeng, T. Durakiewicz, L. Balicas, H. Lin, A. Bansil, S. Shin, Z. Fisk, and M. Z. Hasan, Surface electronic structure of the topological Kondo-insulator candidate correlated electron system $\mathrm{SmB}_{6}$, Nat. Commun. 4, 2991 (2013).

[29] Y. Ishida, T. Otsu, T. Shimada, M. Okawa, Y. Kobayashi, F. Iga, T. Takabatake, and S. Shin, Emergent photovoltage on $\mathrm{SmB}_{6}(001)$ surface upon bulk-gap evolution revealed by pump-and-probe photoemission spectroscopy, Sci. Rep. 5, 8160 (2015).

[30] D. J. Kim, S. Thomas, T. Grant, J. Botimer, Z. Fisk, and J. Xia, Surface Hall effect and nonlocal transport in $\mathrm{SmB}_{6}$ : Evidence for surface conduction, Sci. Rep. 3, 3150 (2013).

[31] S. Wolgast, C. Kurdak, K. Sun, J. W. Allen, D.-J. Kim, and Z. Fisk, Low-temperature surface conduction in the Kondo insulator $\mathrm{SmB}_{6}$, Phys. Rev. B 88, 180405(R) (2013).

[32] S. Wolgast, Y. S. Eo, T. Öztürk, G. Li, Z. Xiang, C. Tinsman, T. Asaba, B. Lawson, F. Yu, J. W. Allen, K. Sun, L. Li, C. Kurdak, D.-J. Kim, and Z. Fisk, Magnetotransport measurements of the surface states of samarium hexaboride using Corbino structures, Phys. Rev. B 92, 115110 (2015).

[33] P. Syers, D. Kim, M. S. Fuhrer, and J. Paglione, Tuning Bulk and Surface Conduction in the Proposed Topological Kondo Insulator $\mathrm{SmB}_{6}$, Phys. Rev. Lett. 114, 096601 (2015).

[34] S. Biswas, M. Ciomaga Hatnean, G. Balakrishnan, and A. Bid, Probing the interplay between surface and bulk states in the topological Kondo insulator $\mathrm{SmB}_{6}$ through conductance fluctuation spectroscopy, Phys. Rev. B 95, 205403 (2017).

[35] Y. S. Eo, K. Sun, C. Kurdak, D.-J. Kim, and Z. Fisk, Inverted Resistance Measurements as a Method for Characterizing the Bulk and Surface Conductivities of Three-Dimensional Topological Insulators, Phys. Rev. Appl. 9, 044006 (2018).

[36] Y. S. Eo, A. Rakoski, J. Lucien, D. Mihaliov, C. Kurdak, P. F. S. Rosa, and Z. Fisk, Transport gap in $\mathrm{SmB}_{6}$ protected against disorder, Proc. Natl. Acad. Sci. USA 116, 12638 (2019).

[37] W. T. Fuhrman, J. C. Leiner, J. W. Freeland, M. van Veenendaal, S. M. Koohpayeh, W. A. Phelan, T. M. McQueen, and 
C. Broholm, Magnetic dichroism in the Kondo insulator $\mathrm{SmB}_{6}$, Phys. Rev. B 99, 020401(R) (2019).

[38] Y. S. Eo, A. Rakoski, S. Sinha, D. Mihaliov, W. T. Fuhrman, S. R. Saha, P. F. S. Rosa, Z. Fisk, M. C. Hatnean, G. Balakrishnan, J. R. Chamorro, W. A. Phelan, S. M. Koohpayeh, T. M. McQueen, B. Kang, M. S. Song, B. Cho, M. S. Fuhrer, J. Paglione, and C. Kurdak, Bulk transport paths through defects in floating zone and $\mathrm{Al}$ flux grown $\mathrm{SmB}_{6}$, Phys. Rev. Materials 5, 055001 (2021).

[39] Y. S. Eo, S. Wolgast, A. Rakoski, D. Mihaliov, B. Y. Kang, M. S. Song, B. K. Cho, M. C. Hatnean, G. Balakrishnan, Z. Fisk, S. R. Saha, X. Wang, J. Paglione, and C. Kurdak, Comprehensive surface magnetotransport study of $\mathrm{SmB}_{6}$, Phys. Rev. B 101, 155109 (2020).

[40] W. A. Phelan, S. M. Koohpayeh, P. Cottingham, J. A. Tutmaher, J. C. Leiner, M. D. Lumsden, C. M. Lavelle, X. P. Wang, C. Hoffmann, M. A. Siegler, N. Haldolaarachchige, D. P. Young, and T. M. McQueen, On the chemistry and physical properties of flux and floating zone grown $\mathrm{SmB}_{6}$ single crystals, Sci. Rep. 6, 20860 (2016).

[41] S. M. Thomas, X. Ding, F. Ronning, V. Zapf, J. D. Thompson, Z. Fisk, J. Xia, and P. F. S. Rosa, Quantum Oscillations in FluxGrown $\mathrm{SmB}_{6}$ with Embedded Aluminum, Phys. Rev. Lett. 122, 166401 (2019).

[42] S. Gheidi, K. Akintola, K. S. Akella, A. M. Côté, S. R. Dunsiger, C. Broholm, W. T. Fuhrman, S. R. Saha, J. Paglione, and J. E. Sonier, Intrinsic Low-Temperature Magnetism in $\mathrm{SmB}_{6}$, Phys. Rev. Lett. 123, 197203 (2019).

[43] P. F. S. Rosa and Z. Fisk, Bulk and surface properties of $\mathrm{SmB}_{6}$, in Rare-Earth Borides, edited by D. S. Inosov (Jenny Stanford Publishing, Singapore, 2021).

[44] P. F. S. Rosa and Z. Fisk, Flux methods for growth of intermetallic single crystals, in Crystal Growth of Intermetallics, edited by P. Gille and Y. Grin (De Gruyter, Berlin, 2018), pp. 49-60.

[45] J. C. Souza, M. König, M. V. Ale Crivillero, M. O. Malcolms, D. Ehlers, R. R. Urbano, Z. Fisk, P. F. S. Rosa, P. G. Pagliuso, S. Wirth, and J. Sichelschmidt, Surface excitations relaxation in a Kondo insulator, Phys. Rev. Research (to be published).

[46] M. Hartstein, H. W. Toews, Y.-T. Hsu, B. Zeng, X. Chen, M. Ciomaga Hatnean, Q. R. Zhang, S. Nakamura, A. S. Padgett, G. Rodway-Gant, J. Berk, M. K. Kingston, G. H. Zhang, M. K. Chan, S. Yamashita, T. Sakakibara, Y. Takano, J.-H. Park, L. Balicas, N. Harrison et al., Fermi surface in the absence of a Fermi liquid in the Kondo insulator $\mathrm{SmB}_{6}$, Nat. Phys. 14, 166 (2018).

[47] K. Flachbart, K. Gloos, E. Konovalova, Y. Paderno, M. Reiffers, P. Samuely, and P. Svec, Energy gap of intermediate-valent
$\mathrm{SmB}_{6}$ studied by point-contact spectroscopy, Phys. Rev. B 64, 085104 (2001).

[48] J. C. Souza, P. F. S. Rosa, J. Sichelschmidt, M. Carlone, P. A. Venegas, M. O. Malcolms, P. M. Menegasso, R. R. Urbano, Z. Fisk, and P. G. Pagliuso, Metallic islands in the Kondo insulator $\mathrm{SmB}_{6}$, Phys. Rev. Research 2, 043181 (2020).

[49] S. Lee, X. Zhang, Y. Liang, S. W. Fackler, J. Yong, X. Wang, J. Paglione, R. L. Greene, and I. Takeuchi, Observation of the Superconducting Proximity Effect in the Surface State of $\mathrm{SmB}_{6}$ Thin Films, Phys. Rev. X 6, 031031 (2016).

[50] K. Flachbart, S. Gabáni, E. Konovalova, Y. Paderno, and V. Pavlík, Ground state formation in intermediate valent $\mathrm{SmB}_{6}$, Physica B 293, 417 (2001).

[51] W. A. Phelan, S. M. Koohpayeh, P. Cottingham, J. W. Freeland, J. C. Leiner, C. L. Broholm, and T. M. McQueen, Correlation Between Bulk Thermodynamic Measurements and the Low-Temperature-Resistance Plateau in $\mathrm{SmB}_{6}$, Phys. Rev. X 4, 031012 (2014).

[52] B. Y. Kang, C.-H. Min, S. S. Lee, M. S. Song, K. K. Cho, and B. K. Cho, Magnetic and nonmagnetic doping dependence of the conducting surface states in $\mathrm{SmB}_{6}$, Phys. Rev. B 94, 165102 (2016).

[53] M. Orendáč, S. Gabáni, G. Pristáš, E. Gažo, P. Diko, P. Farkašovský, A. Levchenko, N. Shitsevalova, and K. Flachbart, Isosbestic points in doped $\mathrm{SmB}_{6}$ as features of universality and property tuning, Phys. Rev. B 96, 115101 (2017).

[54] S. V. Demishev, M. A. Anisimov, V. V. Voronov, M. I. Gilmanov, V. V. Glushkov, M. S. Karasev, V. B. Filipov, and N. Y. Shitsevalov, Surface conductivity of the ytterbium-doped topological Kondo-insulator $\mathrm{SmB}_{6}$, Dokl. Phys. 65, 233 (2020).

[55] N. Wakeham, Y. Q. Wang, Z. Fisk, F. Ronning, and J. D. Thompson, Surface state reconstruction in ion-damaged $\mathrm{SmB}_{6}$, Phys. Rev. B 91, 085107 (2015).

[56] P. J. W. Moll, Focused ion beam microstructuring of quantum matter, Annu. Rev. Condens. Matter Phys. 9, 147 (2018).

[57] T. Miyamachi, S. Suga, M. Ellguth, C. Tusche, C. M. Schneider, F. Iga, and F. Komori, Evidence for in-gap surface states on the single phase $\mathrm{SmB}_{6}(001)$ surface, Sci. Rep. 7, 12837 (2017).

[58] S. Sen, N. S. Vidhyadhiraja, E. Miranda, V. Dobrosavljević, and W. Ku, Fragility of the Kondo insulating gap against disorder: Relevance to recent puzzles in topological Kondo insulators, Phys. Rev. Research 2, 033370 (2020).

[59] M. Abele, X. Yuan, and P. S. Riseborough, Topological nonmagnetic impurity states in topological Kondo insulators, Phys. Rev. B 101, 094101 (2020).

[60] V. Sacksteder, T. Ohtsuki, and K. Kobayashi, Modification and Control of Topological Insulator Surface States Using Surface Disorder, Phys. Rev. Appl. 3, 064006 (2015). 